\title{
The pure rotational spectrum of thorium monosulfide, ThS.
}

\author{
Timothy C. Steimle and Ruohan Zhang \\ Department of Chemistry and Biochemistry \\ Arizona State University \\ Tempe, Arizona, 85287-1604, U.S.A.
}

Michael C. Heaven

Department of Chemistry

Emory University

Atlanta, GA 30322, U.S.A.

\author{
Suggested Running Head: "Microwave spectrum of ThS" \\ Phone Numbers: (480) 965-3265 (voice), (480) 965-2747 (FAX) \\ Number of Tables: 1 \\ Number of Figures: 2 \\ Prepared for: Chem. Phys. Letts. \\ ${ }^{\dagger}$ Corresponding author Prof. T. C. Steimle, Department of Chemistry and Biochemistry, \\ Arizona State University, Tempe, Arizona 85287-1604
}

\footnotetext{
(C) 2015. This manuscript version is made available under the Elsevier user license http://www.elsevier.com/open-access/userlicense/1.0/
} 


\section{Introduction}

Thorium monosulfide (ThS) is an ideal model system for studies of the interactions between an actinide and a soft donor ligand. The spectroscopy and reaction dynamics of gas phase ThS have been examined in recent years [1-4]. Pereira et al. [4] used mass spectrometry to examine the reactions of both ThS and $\mathrm{ThS}^{+}$with $\mathrm{NO}, \mathrm{CO}_{2}$, OCS and $\mathrm{CH}_{2} \mathrm{O}$. The ThS bond dissociation energy and the ionization energy were derived from these measurements. In addition, Liang et al. [3] and Pereira et al. [4] predicted the properties of ThS using Density Functional Theory (DFT) methods. These calculations yielded results that were in good agreement with their experimental results.

To date, the spectroscopic characterization of gas-phase ThS has been quite limited [1, 2]. The electronic spectrum in the $17500-24000 \mathrm{~cm}^{-1}$ spectral range was recorded at medium resolution $\left(\sim 0.15 \mathrm{~cm}^{-1}\right)$ using laser induced fluorescence (LIF). Mass-selected resonance enhanced multi-photonionization was used to confirm the attribution of specific LIF bands to ThS and dispersed LIF spectrum was used to determine the vibrational frequency for $X^{1} \Sigma^{+}$, and the term energies for the $a^{3} \Delta_{1}$ and $a^{3} \Delta_{2}$ states[1]. In this study, $a b$ initio and DFT calculations were also used to predict the properties of the low-energy electronic states of ThS. Multireference complete active space calculations indicated that the ground state has appreciable multi-reference character (primarily an admixture of the $\operatorname{Th}^{2+}\left(7 \mathrm{~s}^{2}\right) \mathrm{S}^{2-}\left(3 \mathrm{~s}^{2} 3 \mathrm{p}^{6}\right)$ and $\mathrm{Th}^{+}\left(7 \mathrm{~s} \sigma^{2} 6 \mathrm{~d} \pi\right) \mathrm{S}^{-}$ $\left(3 s^{2} 3 p \sigma^{2} 3 p \pi^{3}\right)$ configurations). However, DFT calculations yielded good agreement with the spectroscopic data, despite the fact that the methods used were inherently single-reference [1]. 
The prediction of the permanent electric dipole moment, $\vec{\mu}_{\mathrm{el}}$, of a molecule provides a sensitive test of the quality of the electronic state wavefunction. Hence, to further probe the validity of the DFT calculations, Le et al. [2] used optical Stark spectroscopy to measure the $\vec{\mu}_{\mathrm{el}}$ of ThS, $X^{1} \Sigma^{+}$. Rotational lines of the $\{18.6\}-X^{1} \Sigma^{+}(\nu=0)$ band were recorded at high resolution $\left(\sim 0.001 \mathrm{~cm}^{-1}\right)$, both field-free and in the presence of a variable static electric field. The spectra were analyzed to determine fine structure parameters and the $\left|\vec{\mu}_{\mathrm{el}}\right|$ for the $\{18.6\}$ and $X^{1} \Sigma^{+}(\nu=0)$ states. A ground state dipole moment of 4.58(10) D was obtained, along with an improved value for the rotational constant, $B_{0}$. The B3LYP/DFT prediction of $\vec{\mu}_{\mathrm{el}}(4.37 \mathrm{D})$ was close to the measured value [2], providing further evidence that the DFT calculation was recovering the ground state properties correctly, rather than an agreement resulting from a fortuitous cancellation of errors.

The spectroscopic studies reported to date have provided a reasonably accurate value for the ThS $X^{1} \Sigma^{+} v=0$ rotational constant. As the experiments were carried out using jet-cooled samples of ThS, the centrifugal constant, which is sensitive to electronic perturbations, could not be accurately determined. However, given the large ground state $\left|\vec{\mu}_{\mathrm{el}}\right|$ of $\mathrm{ThS}$ it is a promising candidate for microwave studies of the pure rotational transitions. Here we report on the recording and analysis of the pure rotational spectrum of $\mathrm{Th}^{32} \mathrm{~S}$ for the $X^{1} \Sigma^{+} v=0$ state using the method of separated field, pump/probe microwave optical double resonance (PPMODR). In addition to obtaining very accurate molecular constants, these measurements demonstrate that the sensitive and versatile PPMODR technique can be readily applied to heavy element compounds over a wide frequency range. Prior to the results reported here, the only actinide 
containing molecules for which rotational transitions had been reported were ThO [5-7] and $\mathrm{UO}[8]$.

\section{Experimental}

The experiments were performed at the Arizona State University. The cold $\left(\mathrm{T}_{\text {rot }}<10 \mathrm{~K}\right)$ pulsed molecular beam production scheme for ThS was identical to that used in the optical Stark measurements [2]. A continuously rotating thorium metal rod was laser-ablated in the presence of a $\mathrm{CS}_{2} / \mathrm{Ar}$ gas mixture, and the products were supersonically expanded into an evacuated chamber. To entrain the $\mathrm{CS}_{2}$ vapor, $\mathrm{Ar}$ gas at a pressure of approximately 600 psi was flowed over the surface of liquid carbon disulfide $\left(\mathrm{CS}_{2}\right)$ held at $0{ }^{\circ} \mathrm{C}$. At $0{ }^{\circ} \mathrm{C}$ the vapor pressure of $\mathrm{CS}_{2}$ is approximately 2 psi. A block diagram of the PPMODR experimental set-up is given in Figure 1 and is similar to that previously described $[9,10]$. In this scheme the molecular sample was sequentially exposed to an intense $(\sim 300 \mathrm{~mW})$ pump laser beam, tunable microwave radiation and, a weak ( $2 \mathrm{~mW})$ probe laser beam. The pump beam was double-passed by reflection. The microwave induced rotational transition was monitored as an increase in the probe beam LIF signal. The optical pumping and probing radiation were the same frequency and tuned to various $Q$-branch features of the $\{18.6\}-X^{1} \Sigma^{+}(v=0)$ band. To minimize the noise associated with scattered laser light, the resulting probe beam LIF was viewed off-resonance through a $680 \pm 10$ $\mathrm{nm}$ band-pass filter that coincides with the $\{18.6\} \rightarrow a^{3} \Delta_{1}$ emission wavelength. The filtered LIF signal was detected with a cooled photo-multiplier tube and processed using photon-counting techniques. Tunable microwave radiation was generated using an active solid state multiplier and frequency synthesizer operating in the $13.1-19.6 \mathrm{GHz}$ range. $\mathrm{A} \times 4$ multiplier was used for frequencies below $80 \mathrm{GHz}$ and a $\times 5$ multiplier was used for frequencies above $80 \mathrm{GHz}$. A rubidium frequency standard was used to generate the time-base for the frequency synthesizer. 
The stabilized microwave radiation was introduced into the chamber via a homemade $26^{\circ}$ " $\mathrm{H}-$ plane" sectoral horn antenna (approximately $3 \mathrm{~cm}$ "H-field" $\times 0.4 \mathrm{~cm}$ "E-field") for the 50-75 $\mathrm{GHz}$ radiation, and a standard gain, E-band, pyramidal horn (approximately $3.0 \mathrm{~cm}$ "H-field" $\times$ $2.3 \mathrm{~cm}$ "E-field") for the $75-98 \mathrm{GHz}$ radiation.

\section{Observation}

The $J^{\prime \prime}=7 \rightarrow J^{\prime}=8$ through $J^{\prime \prime}=14 \rightarrow J^{\prime}=15$ pure rotational transitions of the ThS $X^{1} \Sigma^{+}(\nu=0)$ state were measured using the $Q$-branch lines of the $\{18.6\}-X^{1} \Sigma^{+}(v=0)$ band for optical pumping and probing. The field-free LIF spectrum in the region of the low- $J$-branch features can be found in Ref. [2]. The observed spectrum for the $J^{\prime \prime}=13 \rightarrow J^{\prime}=14$ rotational transition obtained by pumping and probing the $Q(14)\left(v=18258.7720 \mathrm{~cm}^{-1}\right)$ line, and the associated energy levels, are presented in Figure 2. The spectrum represents the sum of twelve scans with an average of 20 ablation pulses at a given microwave frequency. The microwave synthesizer was stepped in $1 \mathrm{kHz}$ increments, resulting in $6 \mathrm{kHz}$ increments from the active multiplier. In the absence of the pump beam the LIF signal was approximately 350 counts/20 ablation pulses, which was reduced to approximately 160 counts/20 ablation pulses in the presence of the pump beam. For the microwave power used to record the spectrum shown in Figure 2, an increase of approximately 80 counts $/ 20$ ablation pulses was observed when the microwave frequency was tuned to resonance. The line width of the $J^{\prime \prime}=13 \rightarrow J^{\prime}=14$ transition was approximately $25 \mathrm{kHz}$ for the full width at half maximum (FWHM) and this was very sensitive to the microwave power. The spectrum in Figure 2 was recorded using $30 \mathrm{db}$ attenuation of the approximate 5 $\mathrm{mW}$ radiation generated by the active multiplier (i.e. approximately $5 \mu \mathrm{W}$ ). Using the $3.0 \mathrm{~cm} \times$ $2.3 \mathrm{~cm}$ dimensions of the E-band pyramidal horn, the intensity was estimated to be approximately $0.5 \mu \mathrm{W} / \mathrm{cm}^{2}$. 
The observed transition frequencies, which are listed in Table 1, were modeled using standard expression for a molecule with no nuclear spin in a ${ }^{1} \Sigma$ state:

$$
v=2 B_{0} J^{\prime}-4 D_{0} J^{\prime 3}
$$

Linear least squares fitting yielded values of $B_{0}=3275.05033 \pm 0.00041$ and $D_{0}=0.0006699 \pm$ $0.0000012 \mathrm{MHz}$ with a correlation coefficient 0.9511. Frequencies calculated using these parameters are also presented in Table 1 . The standard deviation of the fit was $7 \mathrm{kHz}$, which is consistent with the estimated measurement uncertainty.

\section{Discussion}

The bond length, $r_{0}$, derived from the rotational constant $B_{0}$ is $2.343392 \AA$. The rotational constant from the microwave spectrum is $0.6817 \mathrm{MHz}$ greater than the constant derived from the high-resolution optical study of the $\{18.6\}-X^{1} \Sigma^{+}$transition. The difference is within the 0.96 $\mathrm{MHz}, 1 \sigma$, error range of the former determination [2]. It is of interest to compare the value for centrifugal distortion constant with the estimate derived from the Kratzer relationship [11], $D_{e}=4 B_{e}^{3} / \omega_{e}^{2}$. Using the present value for $B_{0}$ to approximate $B_{e}$, and the vibrational constant from Ref. [1] $\left(\omega_{e}=479 \mathrm{~cm}^{-1}\right)$ yields a value for $D_{e}$ of $0.00068 \mathrm{MHz}$. Similarly, application of the Kratzer relationship to the ground state of ThO using the data of Ref. [12] gives a $D_{e}$ value of $0.0055 \mathrm{MHz}$, that coincides with the experimental value. This model works well for both $\mathrm{ThO}$ and $\mathrm{ThS}$ because the ground states are unperturbed. Their low-lying electronic states do not have the correct symmetry to interact with the ground state. In contrast, the low-lying states of UO are strongly interacting [13], such that the Kratzer relationship does not, and is not expected to give meaningful estimates. 
Molecular constants for ThS $X^{1} \Sigma^{+}$were also derived from the B3LYP/DFT potential energy curve described in Ref. [2]. Values for constants, obtained using the program Level 8.0 [14] to solve for the ro-vibrational eigenstates, were $B_{0}=3268.9$ and $D_{0}=0.000666 \mathrm{MHz}$. The near agreement with the experimental values provides further support for the assumption that computationally inexpensive DFT calculations can yield meaningful predictions and insights concerning the structure and bonding of simple actinide compounds (i.e., those that do not contain actinide metal-metal bonds).

Lastly, after some years of studying actinide species in the gas phase, we find that laser ablation produces oxides are far more readily than any other compound. Hence, it is no surprise to find that the first compounds that have been characterized using microwave spectroscopy are ThO and UO. The present study shows that the PPMODR technique has the sensitivity to provide high-quality spectra for a sulfide, and will be suitable for future studies of actinide chalcogenides, halides and pnictides.

\section{ACKNOWLEDGMENTS}

This research has been supported at ASU by a National Science Foundation, Division of Chemistry grant (CHE-1265885). The work at Emory University is supported by the US Department of Energy under grant DE-FG02-01ER15153-12. 


\section{References}

1. J.H. Bartlett, I.O. Antonov, M.C. Heaven, J. Phys. Chem. A, 117 (2013) 12042-12048.

2. A. Le, M.C. Heaven, T.C. Steimle, J. Chem. Phys., 140 (2014) 024307/024301024307/024305.

3. B. Liang, L. Andrews, J. Phys. Chem. A, 106 (2002) 4038-4041.

4. C.C.L. Pereira, C.J. Marsden, J. Marcalo, J.K. Gibson, Phys. Chem. Chem. Phys., 13 (2011) 12940-12958.

5. C.T. Dewberry, K.C. Etchison, S.A. Cooke, Phys. Chem. Chem. Phys. 9 (2007) 48954897.

6. C.T. Dewberry, K.C. Etchison, G.S. Grubbs, R.A. Powoski, M.M. Serafin, S.A. Peebles, S.A. Cooke, Phys. Chem. Chem. Phys. 9 (2007) 5897-5901.

7. B.E. Long, S.E. Novick, S.A. Cooke, J. Mol. Spectrosc. 302 (2014) 1-2.

8. J. Holt, F.N. Neese, F.C. De Lucia, I. Medvedev, M.C. Heaven, The submillimeter wave spectrum of UO, 69th International Symposium on Molecular Spectroscopy, University of Illinois, 2014. https://dx.doi.org/10.15278/isms.2014.TK11

9. C. Qin, R. Zhang, F. Wang, T.C. Steimle, Chem. Phys. Lett., 535 (2012) 40-43.

10. F. Wang, T.C. Steimle, J. Chem. Phys., 136 (2012) 044312/044311-044312/044316.

11. G. Herzberg, Molecular Spectra and Molecular Structure, Van Nostrand, New York, 1950.

12. G. Edvinsson, A. Lagerqvist, Physica Scripta, 30 (1984) 309-320.

13. L.A. Kaledin, J.E. McCord, M.C. Heaven, J J.H. Bartlett, I.O. Antonov, M.C. Heaven, J. Phys. Chem. A, 117 (2013) 12042-12048. 
14. LeRoy, R. J. LEVEL 8.0: A Computer Program for Solving the Radial Schrödinger Equation for Bound and Quasibound Levels, University of Waterloo Chemical Physics Research Report CP-663 (2007); see http://leroy.uwaterloo.ca/programs/. 
Table 1: Observed and calculated frequencies for the $X^{1} \Sigma^{+}(v=0)$ pure rotational transitions.

\begin{tabular}{|c|l|c|}
\hline \hline \multicolumn{3}{|c|}{ ThS } \\
\hline$J^{\prime \prime} \rightarrow J^{\prime}$ & Observed $^{a}$ & Diff. $^{\mathrm{b}}$ \\
\hline $7 \rightarrow 8$ & 52399.424 & -0.009 \\
\hline $8 \rightarrow 9$ & 58948.960 & 0.008 \\
\hline $9 \rightarrow 10$ & 65498.320 & -0.007 \\
\hline $10 \rightarrow 11$ & 72047.546 & 0.006 \\
\hline $11 \rightarrow 12$ & 78596.580 & 0.003 \\
\hline $12 \rightarrow 13$ & 85145.424 & 0.003 \\
\hline $13 \rightarrow 14$ & 91694.052 & -0.004 \\
\hline $14 \rightarrow 15$ & 98242.464 & -0.001 \\
\hline \multicolumn{3}{|c|}{ Std. dev. $=0.007$} \\
\hline \hline
\end{tabular}

${ }^{a}$ All units are $\mathrm{MHz}$.

${ }^{\mathrm{b}}$ Diff.=Obs.-Calc. (in MHz) obtained using optimized parameters. 
Figure Captions

Figure 1. A block diagram of the PPMODR spectrometer.

Figure 2. The $J^{\prime \prime}=13 \rightarrow J^{\prime}=14$ rotational transitions of the $X^{1} \Sigma^{+}(v=0)$ state of $\mathrm{ThS}$ recorded by PPMODR. Conditions; pump beam $200 \mathrm{~mW}$, double passed, probe beam $2 \mathrm{~mW}$. Microwave intensity $0.5 \mu \mathrm{W} / \mathrm{cm}^{2}$. 
Figure 1

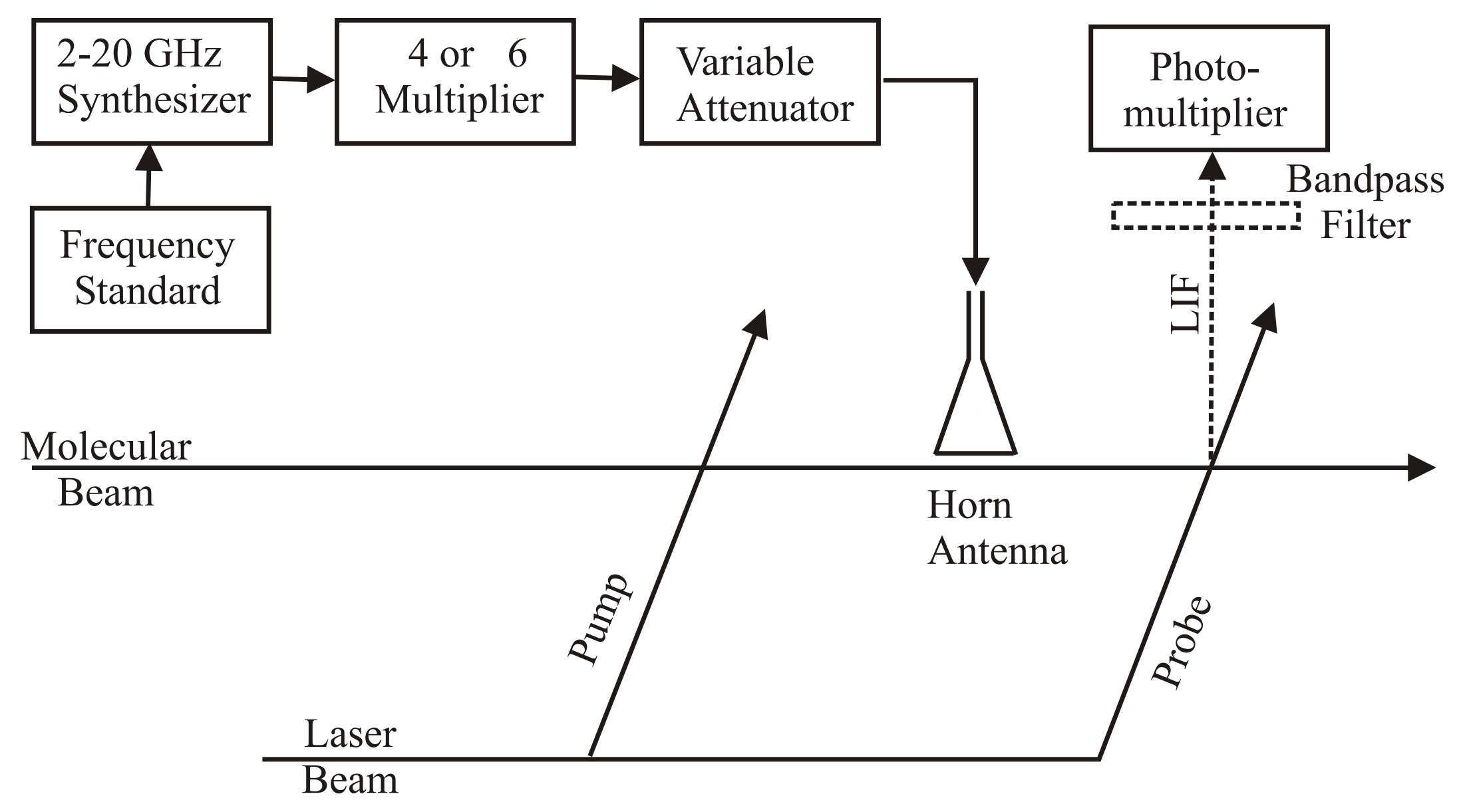


Figure 2

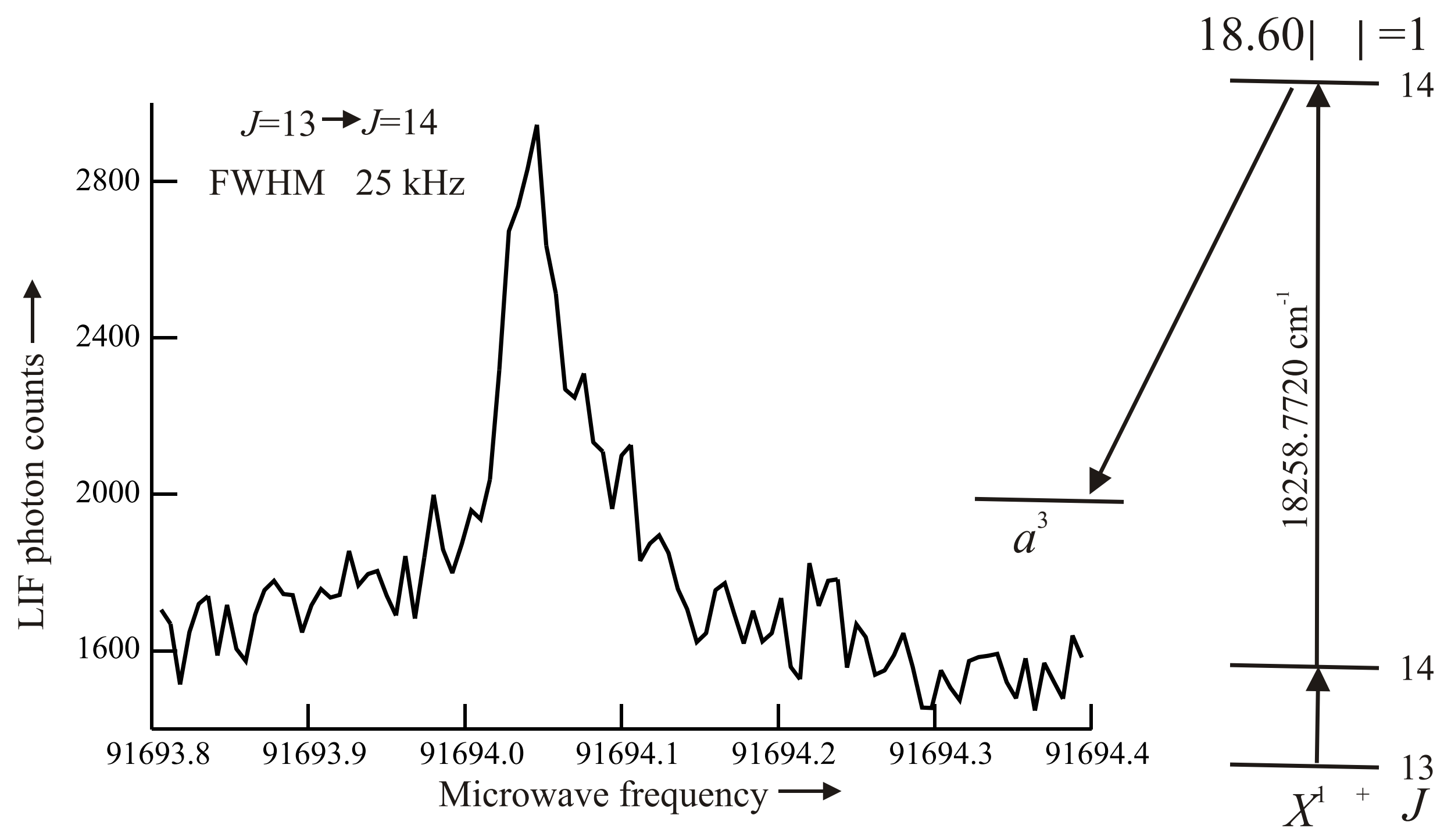




\section{$2.3434 \AA$}
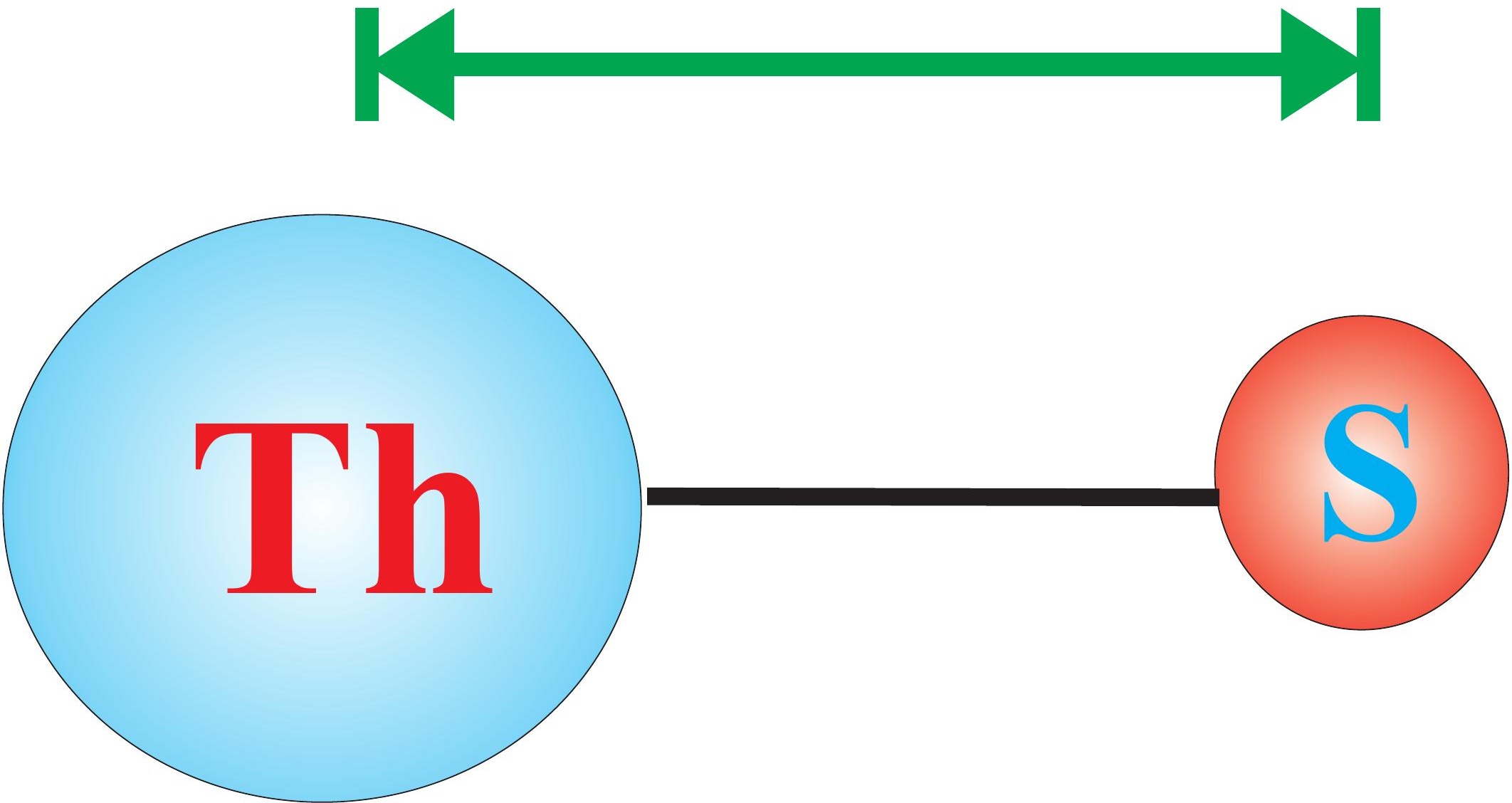